\title{
Genética e fisiopatologia dos transtornos depressivos
}

\author{
Beny Lafer ${ }^{1}$ e Homero Pinto Vallada Filho ${ }^{1}$
}

\section{Resumo}

Fatores genéticos, neurobiológicos e ambientais participam da gênese das depressões. Esta breve revisão visa enfatizar os estudos sobre os aspectos genéticos, neuroquímicos e neuroanatômicos na etiologia e fisiopatologia das depressões e suas implicações no desenvolvimento de novos tratamentos. Procura-se enfatizar as limitações encontradas até o momento na tentativa do estabelecimento de uma etiopatogenia comum às depressões, principalmente em função da dificuldade no diagnóstico e da heterogeneidade na fenomenologia do episódio agudo e no curso longitudinal. Perspectivas para futuras pesquisas também são apresentadas.

\section{Descritores}

Transtornos depressivos; depressão; etiologia; fisiopatologia; genética; neuroimagem; tratamento

\begin{abstract}
Genetic, neurobiological and environmental factors play a role in the pathogenesis of depressive disorders. This brief review points to the major studies on the genetics, neurochemistry and neuroanatomy of depressive disorders as well as their implications on the development of new treatments. The limitations found by several groups in establishing a common etiopathogenesis are emphasised in light of the difficulty in establishing a reliable diagnosis and of the clinical heterogeneity found in the phenomenology of acute episodes and long-term outcome. Future research perspectives are also presented.
\end{abstract}

\section{Keywords}

Depressive disorders; depression; etiology; pathophysiology; genetics; brain imaging; treatment

\section{Genética da depressão}

Observações feitas ainda no século XIX por psiquiatras europeus como Morel, Griesinger, Maudsley e Kraepelin já davam conta que esse distúrbio afetivo parecia se concentrar mais em determinadas famílias. ${ }^{1}$ Portanto, desde essas observações clínicas, tem-se procurado demonstrar a existência de um componente genético para as depressões.

\section{O Problema do fenótipo}

Quando se pretende investigar o componente genético da depressão, uma das dificuldades centrais é a caracterização precisa do fenótipo. Chamamos de fenótipo aquelas características observáveis num indivíduo que pretendemos relacionar a uma determinada constituição genética. No caso específico da depressão, mesmo com os critérios operacionais atuais, a confiabilidade do diagnóstico entre diferentes entrevistadores varia bastante, dependendo do número de episódios e da gravidade. Assim, episódios depressivos leves recorrentes têm baixa confiabilidade (kappa $=0,37$ ), enquanto um episódio único e grave tem boa confiabilidade (kappa $=0,72$ ). Além disso, há relatos que mostram que a confiabilidade do diagnóstico de um episódio depressivo num período superior a um ano em populações não-clínicas é muito baixa (kappa $=0,34){ }^{2}$ Podemos imaginar, portanto, que diferenças na conceituação do transtorno podem determinar alterações significativas nos resultados de investigações genéticas.

\section{Estudos genético-epidemiológicos}

\section{Estudos com famílias}

No que se refere aos estudos de família na depressão, nos últimos trinta anos, tem-se, em regra, seguido a classificação sugerida por Leonhard. ${ }^{3}$ Essa classificação subdivide as alterações do humor em distúrbio bipolar - em que encontramos mania e depressão - e distúrbio depressivo unipolar - em que só encontramos episódios de depressão.

Angst, em 1966 na Suíça, e Perris, no mesmo ano, na Suécia, independentemente, apresentaram os primeiros resultados de estudos sistemáticos em famílias, utilizando a subdivisão de Leonhard. Ambos demonstraram agregação familiar das alterações do humor e, mais ainda, validaram a subdivisão da psicose maníaco-depressiva, encontrando maior freqüência de bipolares entre os familiares de bipolares e de unipolares entre os parentes de depressivos unipolares. A maioria dos estudos que se seguiram concordam com esses autores tanto na agregação familiar quanto na subdivisão. Os resultados desses estudos em famílias de pacientes com transtorno do humor podem ser resumidos assim:

1. Risco de parentes em primeiro grau de indivíduos nãoafetados, representativos da população geral, é de quase $1 \%$ 
para doença bipolar e de cerca de 5\% para depressão unipolar.

2. Quando comparamos este risco com o dos parentes em primeiro grau de pacientes com depressão observamos que o risco para doença depressiva unipolar está aumentado em aproximadamente três vezes, enquanto o risco de transtorno bipolar nestes familiares é quase o mesmo da população geral.

3. Finalmente, quando comparamos o grupo de familiares de indivíduos da população geral com o grupo de parentes em primeiro grau de portadores de transtorno bipolar, verificamos que o risco para a depressão unipolar está aumentado em três vezes, enquanto o risco para doença bipolar nestes parentes está aumentado em cerca de sete vezes.

\section{Estudos com gêmeos}

Este tipo de estudo parte da premissa de que gêmeos monozigóticos (MZ) e dizigóticos (DZ) sofrem influência ambiental semelhante, contudo, os MZ são geneticamente idênticos, enquanto os DZ compartilham apenas a metade de sua carga genética. Desse modo, em enfermidades determinadas pelo ambiente a concordância entre $\mathrm{MZ}$ e DZ seria próxima, ao passo que em enfermidades genéticas a concordância nos MZ seria significativamente maior que nos DZ e tenderia a se aproximar de $100 \%$.

Entre os estudos mais importantes com gêmeos na depressão podemos citar os seguintes: Bertelsen et al investigaram na Dinamarca um total de 123 pares de gêmeos, dos quais um dos irmãos já apresentava transtorno afetivo do humor. Esses autores observaram uma concordância com relação à doença bipolar de $79 \%$ para MZ e de $19 \%$ para DZ, enquanto na depressão unipolar as taxas de concordância de MZ e DZ foram de $64 \%$ e $24 \%$, respectivamente. ${ }^{4}$ Em uma amostra norueguesa de probandos predominantemente unipolares, Torgersen encontrou uma taxa de concordância de $51 \%$ para MZ e de $20 \%$ em DZ. ${ }^{5}$ McGuffin et al investigaram na Inglaterra pares de gêmeos, em sua maioria depressivos unipolares, e observaram uma concordância entre $\mathrm{MZ}$ de $53 \%$ e em DZ de $28 \% .^{6} \mathrm{O}$ estudo mais recente é o de Kendler et al, que verificou, em uma amostra de gêmeos suecos, uma concordância de $69 \%$ em pares MZ e de $34 \%$ em pares DZ. ${ }^{2}$ Podemos concluir, com base nesses dados, que a taxa de concordância para depressão em gêmeos $\mathrm{MZ}$ é duas a três vezes maior do que nos DZ, reforçando a hipótese de um componente genético nesta enfermidade.

\section{Estudos com adotados}

No intuito de separar por completo a influência do ambiente da influência do componente genético, utiliza-se a estratégia de investigação da doença em adotados. Estes estudos têm basicamente dois desenhos principais. No primeiro, investiga-se a freqüência da enfermidade entre os pais biológicos e os pais adotivos de crianças adotadas que vieram a desenvolver a doença. No segundo, observa-se a freqüência do distúrbio nos filhos biológicos de portadores da doença que foram adotados ao nascimento, e compara-se com filhos biológicos de pais saudáveis que também foram adotados logo após o nascimento. Naturalmente esses estudos são muito difíceis de serem executados não só pelas questões éticas que eles envolvem, mas também porque exigem bons serviços de registros de adoções, que, até o presente, encontramos predominantemente nos países escandinavos.
Com relação à doença depressiva, podemos identificar apenas quatro relatos de investigações com adotados. O primeiro estudo foi conduzido na Bélgica por Mendlewicz e Rainer, que verificaram 29 adotados com doença maníaco-depressiva e observaram uma prevalência de distúrbios afetivos em $28 \%$ dos pais biológicos desses indivíduos, comparado a uma prevalência de $12 \%$ nos pais adotivos. ${ }^{7}$ Um segundo estudo foi conduzido por Cadoret, que, numa amostra de mães com transtorno afetivo, evidenciou uma freqüência seis vezes maior de depressão em seus filhos adotados ao nascer, quando comparada aos filhos de mães com outras condições clínicas também adotados no nascimento. ${ }^{8}$ Um estudo com adotados suecos realizado por Von Knorring et al encontrou poucas evidências de um componente genético em pais biológicos de 56 doentes afetivos adotados. ${ }^{9}$ Entretanto, questões metodológicas como a baixa confiabilidade das fichas de seguro-saúde que o autor utilizou em suas investigações podem explicar a discrepância com os demais estudos. Por último Wender et al investigaram uma amostra dinamarquesa de 71 adotados afetados por transtornos do humor e relataram uma prevalência oito vezes maior em casos de depressão unipolar e 15 vezes maior em casos de suicídio nos pais biológicos desses indivíduos, quando comparados a seus pais adotivos. ${ }^{10}$ Concluímos, portanto, que os relatos de estudos com adotados na depressão são, em sua maioria, consistentes com a presença de um componente genético na determinação desta enfermidade.

\section{Estudos de genética molecular Estudos de ligação}

Este tipo de investigação em geral necessita de famílias grandes e com múltiplos afetados. Entretanto, a principal limitação dessa estratégia é o pressuposto de que a doença em estudo seja causada por um único gene. $\mathrm{O}$ que se verifica atualmente é que a depressão não apresenta um padrão de transmissão compatível com um modelo de herança mendeliana simples. Portanto, esse tipo de estudo tem sido muito pouco utilizado.

\section{Estudos de associação}

Uma outra investigação que é realizada na busca de genes causadores de doenças é o estudo de associação. Nesta investigação o pesquisador levanta a hipótese de que um determinado gene esteja envolvido na patofisiologia do transtorno (gene candidato) e, a partir daí, verifica se a freqüência de uma determinada variação ou alteração na estrutura desse gene é significativamente maior entre a população de afetados do que nos não-afetados. Busca-se, portanto, determinar se há uma associação entre a condição de afetado e aquela variação no gene. Este tipo de estratégia apresenta a vantagem de poder detectar genes que apresentam efeitos discretos ou moderados na determinação de uma doença, o que a torna mais adequada nas enfermidades poligênicas e multifatoriais.

Vários genes candidatos têm sido investigados em estudos de associação tanto no distúrbio bipolar quanto na depressão unipolar. Baseados na hipótese de disfunção dos sistemas monoaminérgicos cerebrais, o primeiro gene candidato investigado no transtorno bipolar foi a tirosina hidroxilase, uma enzima que limita o ritmo de síntese das monoaminas. Vários outros estudos, a partir dos anos 90, têm sido realizados sobre 
a depressão. Entretanto, os estudos de associação conduzidos até o presente não foram capazes de estabelecer resultados definitivos, haja visto que a maioria dos achados positivos não puderam ser replicados. O que sugere que pode tratar-se de falso-positivos decorrentes de estratificações populacionais ou do acaso.

\section{Neuroquímica}

Nos últimos 30 anos, a neuroquímica é a área que vem recebendo maior destaque nas pesquisas sobre a fisiopatologia da depressão. Isto teve início a partir do descobrimento do mecanismo de ação dos antidepressivos. Este mecanismo foi estudado inicialmente em relação às alterações agudas sobre os níveis sinápticos dos neurotransmissores na tentativa de estabelecer hipóteses sobre a fisiopatologia dos transtornos do humor. A partir da observação de que essas hipóteses eram muitos limitadas na sua capacidade de explicar a fisiopatologia, foram propostas hipóteses mais complexas, focalizando as alterações em múltiplos sistemas de neurotransmissão e as adaptações celulares e moleculares aos medicamentos antidepressivos. Apresentaremos a seguir um resumo das hipóteses neuroquímicas para a depressão.

\section{Sistemas monoaminérgicos: noradrenalina, serotonina e dopamina}

Os sistemas monoaminérgicos se originam em pequenos núcleos no tronco cerebral e mesencéfalo e projetam-se difusamente pela córtex e sistema límbico. Esses sistemas são compostos por neurônios que contêm norepinefrina (NE), serotonina (5-HT) e dopamina (DA). Junto com a acetilcolina (ACh), eles exercem efeitos de modulação e integração sobre outras atividades corticais e subcorticais e estão envolvidos na regulação da atividade psicomotora, apetite, sono e, provavelmente, do humor.

A observação dos efeitos antidepressivos dos inibidores da monoaminoxidase (MAO) e dos tricíclicos, a partir do final da década de 50, permitiu o surgimento de especulações sobre os substratos cerebrais nos transtornos afetivos. Pesquisas nas áreas básicas sugeriam que os inibidores da MAO aumentavam as concentrações cerebrais de noradrenalina e serotonina. Os antidepressivos tricíclicos, por sua vez, inibiam a recaptura sináptica das monoaminas, principalmente de NE, mas também de 5-HT, aumentando agudamente os níveis sinápticos. Essas e outras observações culminaram em 1965 com a hipótese catecolaminérgica, que propunha a existência de uma depleção de NE no nível sináptico como fator patogenético nos transtornos depressivos. ${ }^{11}$ A hipótese envolvendo a participação da serotonina foi subseqüentemente proposta por Coppen. ${ }^{12}$

Pode-se dizer que a partir da formulação das hipóteses noradrenérgica e serotonérgica, a atividade desses sistemas vem sendo exaustivamente pesquisada. Estudos básicos e clínicos procuraram confirmar a hipótese monoaminérgica na fisiopatologia da depressão e descreveram anormalidades nos níveis desses metabólitos no sangue, urina e líquido céfaloraquidiano (LCR). ${ }^{13}$ Essas pesquisas detectaram diversas alterações nesses sistemas, mas muitas vezes não foram replicadas. ${ }^{14}$

A partir da década de 70 as pesquisas focalizaram mais a complexa interação entre os neurotransmissores. Daí o advento da hipótese permissiva da serotonina, que postulava um efeito modulador do sistema serotonérgico sobre NE e DA. A hipótese permissiva representou um avanço sobre hipóteses anteriores que enfatizavam a falta ou excesso de um só neurotransmissor, e permitiu a integração do conhecimento de que depressão e mania não são estados totalmente opostos, mas que compartilham alguns sintomas e achados biológicos.

Os antidepressivos aumentam os níveis de NE e 5-HT algumas horas após administrados, mas demoram de 2 a 3 semanas para exercer o efeito terapêutico. Foi necessário o estabelecimento de hipóteses que explicassem esta latência na resposta e que levassem em conta os efeitos adaptativos crônicos dos receptores na administração de antidepressivos.

\section{Hipótese da dessensibilização de receptores}

Alterações da função dos sistemas neurotransmissores podem ocorrer através da mudança na sensibilidade de receptores pré e pós-sinápticos, sem alteração da quantidade do próprio neurotransmissor. Essa observação permitiu que a hipótese de deficiência de neurotransmissores fosse modificada e, em seu lugar, proposta a hipótese de dessensibilização dos receptores. Tal hipótese propunha que o atraso no aparecimento do efeito terapêutico dos antidepressivos estava relacionado a alterações no número e sensibilidade dos receptores monoaminérgicos. Como esse efeito demora dias ou semanas para ocorrer, os investigadores propunham que a depressão podia ser explicada por uma supersensitividade de receptores beta-adrenérgicos. ${ }^{15}$ As alterações presentes na sensibilidade e no número de receptores podem ser vistas meramente como marcadores de adaptações crônicas dos neurônios monoaminérgicos ao invés de representarem o mecanismo terapêutico. ${ }^{16}$ Não há dados convincentes de que a regulação de receptores adreshennérgicos ou serotonérgicos seja o único fator responsável pelos efeitos terapêuticos das drogas antidepressivas. A hipótese de dessensibilização tem limitações e não fica claro se a super ou subsensibilização de receptores é apenas um epifenômeno ou é um passo fundamental na ação antidepressiva.

O desafio das pesquisas contemporâneas sobre o mecanismo antidepressivo das medicações é determinar exatamente o que produz a resposta terapêutica. Os principais mecanismos (que estão sendo estudados) incluem a regulação de enzimas (proteínas quinases) e da expressão gênica que contribuem para mudanças na eficácia sináptica. ${ }^{17}$ Para Post, um melhor entendimento desses mecanismos pode inclusive permitir a integração de fatores ambientais e biológicos na fisiopatologia da recorrência dos episódios ao longo do tempo. ${ }^{18}$

\section{Neuroimagem}

Recentes avanços nos métodos de neuroimagem tornaram possível uma ampliação no conhecimento da neurobiologia das doenças mentais, através da visualização de regiões e/ou circuitos envolvidos na fisiopatologia desses transtornos. ${ }^{19}$ Aplicados ao estudo da depressão, vários estudos utilizando as técnicas de neuroimagem estrutural e funcional apontam anormalidades cerebrais como alterações volumétricas, metabólicas e de fluxo sangüíneo global e regional em pacientes 
com transtornos do humor. ${ }^{20}$ Os resultados dos principais estudos, assim como suas implicações, serão discutidos a seguir.

\section{Neuroimagem estrutural}

\section{Tomografia computadorizada (TC)}

O estudo volumétrico das estruturas cerebrais utilizando a TC é difícil de ser realizado, pois essa técnica não permite um bom contraste entre substância branca e cinzenta, impedindo uma visualização precisa de estruturas subcorticais. A maior parte das pesquisas com pacientes deprimidos utilizando TC foi realizada na década de 80 . Os resultados sugerem um aumento dos ventrículos e alargamento dos sulcos corticais em pelo menos algumas populações com transtornos do humor unipolar e bipolar. ${ }^{21-23}$

\section{Imagem por ressonância magnética nuclear (RMN)}

\section{Achados volumétricos com RMN}

Os estudos iniciais com RMN em pacientes com depressão replicaram os achados encontrados com TC quanto ao aumento de ventrículos e discreta atrofia cortical. ${ }^{23,24}$

Trabalhos mais recentes investigaram os volumes da substância branca e cinzenta, dos lobos frontais, lobos temporais e gânglios da base em pacientes com depressão. Diversos grupos detectaram diminuição no volume dessas estruturas em pacientes com depressão, mas nem sempre os achados foram replicados. Em parte, podemos afirmar que essa dificuldade em replicar esses achados se deve à grande heterogeneidade dos grupos estudados, já que a maior parte dos estudos utilizou os critérios de Depressão Maior do DSM-III-R e DSM-IV, que, operacionalmente, determinam um grupo clinicamente heterogêneo. ${ }^{25}$

Os achados dos estudos volumétricos sugerem a participação de estruturas pertencentes aos circuitos que conectam os lobos frontais aos gânglios da base (circuitos córtex-gânglios da base-tálamo-corticais) na fisiopatologia da depressão, principalmente do circuito pré-frontal e do circuito do cíngulo ("circuito límbico").

\section{Hiperintensidades subcorticais em imagens pesadas em T2}

Hiperintensidades subcorticais são imagens que aparecem com aumento do sinal em imagens de ressonância pesadas em T2. Elas podem ser puntiformes, arredondadas, ou aparecer em aglomerados, principalmente na substância branca periventricular, mas também acometendo os gânglios da base e o tálamo. ${ }^{26}$ Os correlatos neuropatológicos dessas imagens foram estudados por alguns grupos que sugerem uma série de alterações, entre elas: gliose, microlesões vasculares, perda de mielina ou aumento de água no espaço perivascular. ${ }^{27,28}$

Diversos grupos detectaram um aumento de hiperintensidades periventriculares e nos gânglios da base em pacientes deprimidos unipolares, quando comparados a controles normais da mesma idade, principalmente nos pacientes idosos e com depressão refratária. ${ }^{29-31}$

A conclusão desses estudos é que as hiperintensidades subcorticais, principalmente nos gânglios da base, podem aumentar a predisposição do indivíduo à manifestação de quadros depressivos. É possível que essas lesões na substância branca estejam interrompendo os fronto-estriatais, contribuindo na etiopatogenia do quadros depressivos e aumentando o risco de resistência ao tratamento farmacológico. ${ }^{32}$

\section{Neuroimagem funcional}

Novas técnicas de neuroimagem funcional permitem mensurações in vivo do fluxo sangüíneo cerebral regional e do metabolismo cerebral. Pelo menos quatro modalidades estão sendo usadas para estudos da função cerebral em pacientes com depressão:

1. Tomografia por emissão de fóton único (Spect);

2. Tomografia por emissão de pósitrons (PET);

3. Espectroscopia por RM (MRS);

4. Imagem funcional por RM (fMRI) (esta técnica tem sido mais utilizada no estudo da regulação do humor em voluntários normais).

Descrevemos, a seguir, os resultados dos estudos utilizando essas técnicas.

\section{Tomografia por emissão de fóton único (Spect)}

A tomografia por emissão de fóton único (Spect) permite o estudo do fluxo sangüíneo regional cerebral através da administração de isótopos radioativos e a obtenção de cintilografias de perfusão. Usando esta técnica, a maioria dos estudos constatou uma redução global no fluxo sangüíneo cortical na depressão maior, principalmente nas regiões frontais. ${ }^{33-35}$

\section{Tomografia por emissão de pósitrons (PET)}

Dois estudos iniciais realizados na Califórnia usando PET documentaram taxas relativamente baixas de metabolização da glicose nos lobos frontais e nos gânglios da base de pacientes deprimidos, quando comparados a controles normais. ${ }^{36,37} \mathrm{Ou}$ tros estudos observaram taxas metabólicas diminuídas no córtex pré-frontal anterolateral esquerdo em pacientes deprimidos..$^{38,39}$

Drevets et al encontraram uma diminuição de fluxo em caudado e aumento de fluxo em amígdala e lobo frontal. O resultado de hiperfluxo frontal não foi encontrado em outros estudos e foi explicado em função das diferentes amostras estudadas. ${ }^{40}$

$\mathrm{O}$ grupo de Londres utilizou o ${ }^{15} \mathrm{O}$-PET para examinar o fluxo sangüíneo cerebral regional e mostrou que os déficits cognitivos em pacientes com depressão melancólica podem estar associados à redução da atividade no córtex pré-frontal anteromedial esquerdo e aumento da atividade no córtex cerebelar. ${ }^{41}$ Este mesmo grupo de pesquisadores detectou correlações entre retardo psicomotor e decréscimo do fluxo sangüíneo no córtex pré-frontal dorsolateral esquerdo. ${ }^{42}$

Recentemente dois grupos vêm enfatizando a participação da região anterior do giro do cíngulo na fisiopatologia da depressão e descreveram uma diminuição do fluxo sangüíneo nessa região. ${ }^{43,44}$

\section{Espectroscopia por ressonância magnética (MRS)}

O método da espectroscopia por ressonância magnética permite avaliar quantitativamente e qualitativamente a presença de núcleos de vários elementos da tabela periódica, que em função de suas propriedades nucleares e, quando presentes em metabólitos, tornam possível obter informações sobre várias etapas da cascata metabólica, do metabolismo energético e de fosfolípides. ${ }^{45}$ Os átomos mais utilizadas em neuropsiquiatria são o hidrogênio e o fósforo (para avaliação metabólica). ${ }^{46}$ 
Estudos utilizando espectroscopia de hidrogênio nos transtornos do humor foram realizados a partir do início da década de 90 , sendo que o mais importante mostrou uma diminuição nos níveis de colina nos gânglios da base em 41 pacientes com depressão. ${ }^{47}$

Utilizando a espectroscopia do fósforo, Kato et al relataram uma diminuição na concentração de fosfocreatina em pacientes gravemente deprimidos, quando comparados com pacientes levemente deprimidos, ${ }^{48}$ No único estudo de espectroscopia de fósforo dos gânglios da base em pacientes com depressão, Moore et al detectaram uma diminuição nos níveis de ATP nos gânglios da base de pacientes com depressão maior, se comparados com controles normais. Este achado é compatível com o hipometabolismo detectado nos estudos com PET. ${ }^{49}$

\section{Conclusões}

Um dos principais desafios nos estudos genéticos da depressão continua sendo a caracterização precisa do fenótipo. Entretanto, mesmo apresentando variações no conceito de depressão, podese concluir a partir de um grande número de investigações que:

- Estudos com famílias, gêmeos e adotados indicam a existência de um componente genético para depressão tanto unipolar como bipolar.

- Estima-se que este componente genético represente cerca de $40 \%$ da susceptibilidade para desenvolver depressão unipolar e $70 \%$ para o transtorno bipolar.

- O modo de transmissão genética permanece não definido, embora as análises de segregação já sugiram que a depressão é provavelmente multifatorial.

- Os estudos de genética molecular não conseguiram ainda identificar um locus gênico específico para a depressão, possivelmente por se tratar de uma enfermidade com heterogeneidade etiológica.

Os estudos neuroquímicos e de neuroimagem combinados com a identificação de genes de vulnerabilidade à doença e a pesquisa sobre a interação entre o cérebro e o meio ambiente continuam sendo a melhor estratégia no entendimento da neurobiologia dos transtornos do humor.

Não se pretende reduzir toda a patogênese dos quadros depressivos a alterações neuroanatômicas, mas com certeza a melhor compreensão da etiopatogenia poderá futuramente auxiliar na detecção precoce, no desenvolvimento de novos tratamentos, assim como na predição da resposta terapêutica.

\section{Referências bibliográficas}

1. Mitchell P, Mackinnon A,Waters B. The genetics of bipolar disorder. Austral. N. Zealand. Journal of Psychiatry 1993;27:560-80.

2. Kendler KS, Pederson N, Johnson L, Neale MC, Mathe AA. A pilot swedish twin study of affective illness, including hospital-and-populationascertained subsamples. Arch Gen Psychiatry 193;50:699-706.

3. Leonhard K. [The classification of endogenous psychoses]. Robins E, editor. Irvington (NY): 1957.

4. Bertelsen A, Harvald B, Hauge M. A Danish twin study of manic-depressive disorders. Br J Psychiatry 1977; 130:330-51.

5. Torgersen S. Genetic factors in moderately severe and mild affective disorders. Arch Gen Psychiatry 1986;43: 222-26.

6. McGuffin P, Katz R. Nature, nurture and depression: a twin Study. Psychol Med 1991;21:229-35.

7. Mendlewicz J, Rainer JD. Adoption study supporting genetic transmission in manic-depressive illness. Nature 1977;268:327-29.

8. Cadoret RJ. Evidence for genetic inheritance of primary affective disorder in adoptees. Am J Psychiatry 1978; 135:463-66.

9. Von Knorring AL, Cloninger CR, Bohman M, Sigvardsson S. An adoption study of depressive disorders and substance abuse. Arch Gen Psychiatry 1983;40:943-50.

10. Wender PH, Kety SS, Rosenthal D, Schulsinger F, Ortmann J, Lunde I. Psychiatric disorders in the biological and adoptive families of adopted individuals with affective disorders. Arch Gen Psychiatry 1986;43:92329.

11. Schildkraut JJ. The catecholamine hypothesis of affective disorders: a review of supporting evidence. Am J Psychiatry 1965;122:509-20.

12. Coppen AJ, Doogan DP. Serotonin and its place in the pathogenesis of depression. J Clin Psychiatry 1988;49 Suppl: 4-11.

13. Potter WZ, Manji HK. Are monoamine metabolites in cerebrospinal fluid worth measuring? Arch Gen Psychiatry 1993;49:653-56.

14. Delgado PL, Price LH, Heninger GR, Charney DS. Neurochemistry. In: Paykel ES, editor. Handbook of affective disorders. 2nd ed. New York: .The Guilford Press; 1992. p. 219-53.

15. Charney DS, Menekes DB, Heninger GR. Receptor sensitivity and the mechanism of action of antidepressant treatment. Arch Gen Psychiatry 1981;38:1160-80.

16. Hyman SE, Nestler EJ. The molecular foundations of psychiatry. Washington, DC: American Psychiatric Press; 1993.

17. Sheng M, Greenberg ME. The regulation and function of c-fos and other immediate early genes in the nervous system. Neuron 1990;4:477-85.
18. Post RM. Transduction of psychosocial stress into the neurobiology of recurrent affective disorder. Am J Psychiatry 1992;149:999-1010.

19. Dolan RJ. Mood disorders and abnormal cingulate cortex. Trends Cogni Sci 1997;1:28.

20. Lafer B, Renshaw PF, Sachs GS. Major depression and the basal ganglia. Psychiatr Clin North Am 1997;20: 885-96.

21. Pearlson GD, Veroff AE. Computerised tomographic scan changes in manic-depressive illness. Lancet 1981;2: 470-71.

22. Andreasen NC, Swayze II V, Flaum M, Alliger R, Cohen G. Ventricular abnormalities in affective disorder: clinical and demographic correlates. Am J Psychiatry 1990;147:893-900.

23. Elkis H, Friedman L, Wise A, Meltzer HY. Meta-analyses of studies of ventricular enlargement and cortical sulcal prominence in mood disorders. Comparisons with controls or patients with schizophrenia. Arch Gen Psychiatry 1995;52:735-46.

24. Swayze II VW, Andreasen NC, Alliger RJ, Ehrhardt JC, Yuh W. Structural brain abnormalities in bipolar affective disorder. Arch Gen Psychiatry 1990; 47:1054-59.

25. Lafer B, Nierenberg AA, Rosenbaum JF, Fava M. Outpatients with DSM-IIIR versus DSM-IV melancholic depression. Compr Psychiatry 1996;37:37-9.

26. Krishnan KR, Hays JC, Blazer DG. MRI-defined vascular depression Am J Psychiatry 1997;154:497-501.

27. Awad IA, Spetzler RF, Hodak JA, Awad CA, Carey R. Incidental subcortical lesions identified on magnetic resonance imaging in the elderly: I. correlation with age and cerebrovascular risk factors. Stroke 1986;17: 1084-97.

28. Chimowitz MI, Estes ML, Furlan AJ, Awad IA. Further observations on the pathology of subcortical lesions identified on magnetic resonance imaging. Arch Neurol 1992;49:747-52.

29. Krishnan KR, Goli V, Ellinwood EH, France RD, Blazer DG, Nemeroff CB. Leukoencephalopathy in patients diagnosed as major depressive. Biol Psychiatry 1988;23:519-22.

30. Coffey CE, Figiel GS, Djang WT, Saunders WB, Weiner RD. White matter hyperintensity on magnetic resonance imaging: clinical and neuroanatomic correlates in the depressed elderly. J Neuropsychiatry Clin Neurosci 1989;1:135-44.

31. Hickie I, Scott E, Mitchell P, Wilhelm K, Austin MP, Bennett B. Subcortical hyperintensities on magnetic resonance imaging: clinical correlates and prognostic significance in patients with severe depression. Biol Psychiatry $1995 ; 37: 151-60$ 
32. Coffey CE, Figiel GS. Neuropsychiatric significance of subcortical encephalomalacia. In: Carrol BJ, Barrett JE, editors. Psychopathology and the brain. New York: Raven Press; 1991. p. 243-64.

33. Sackeim HA, Prohovnik I. Biology of depressive disorders. In: Mann JJ, Kupfer DJ, editors. Brain imaging studies of depressive disorders. New York: Plenum Press ; 1993. p. 205-72.

34. Austin MP, Dougall N, Ross M, Murray C, O'carroll RE, Moffoot A, et al. Single photon emission tomography with $99 \mathrm{mTc}$-exametazime in major depression and the pattern of brain activity underlying the psychotic/ neurotic continuum. J Affect Disord 1992;26:31-44.

35. Mayberg HS, Lewis PJ, Regenold W, Wagner HN. Paralimbic hypoperfusion in unipolar depression. J Nucl Med 1994;35:929-34.

36. Baxter LR, Phelps ME, Mazziotta JC, Schwartz JM, Gerner R, Selin CE, et al. Cerebral metabolic rates for glucose in mood disorders. Arch Gen Psychiatry 1985;42:411-47.

37. Buchsbaum MS, Wu J, Delisi LE, Holcomb HH, Kessler R, Johnson J, et al. Frontal cortex and basal ganglia metabolic rates assessed by positron emission tomography with $\{18 \mathrm{~F}\} 2-$ deoxyglucose in affective illness. J Affect Disord 1986;10:137-52.

38. Baxter LR, Schwartz JM, Phelps ME, Mazziotta JC, Guze BH, Selin CE, et al. Reduction of prefrontal cortex glucose metabolism common to three types of depression. Arch Gen Psychiatry 1989;46:243-50.

39. Martinot JL. Left prefrontal glucose hypometabolism in the depressed state: a confirmation. Am J Psychiatry 1990;147:1313-17.

40. Drevets WC, Videen TO, Price JL, Preskorn SH, Carmichael ST. A functional anatomical study of unipolar depression. J Neurosci 1992;12:3628-41.

41. Bench CJ, Friston KJ, Brown RG, Scott LC, Frackowiak RS, Dolan RJ.
The anatomy of melancholia-focal abnormalities of cerebral blood flow in major depression. Psychol Med 1992;22:607-15.

42. Bench CJ, Friston KJ, Brown RG, Frackowiak RS, Dolan RJ. Regional cerebral blood flow in depression measured by positron emission tomography : the relationship with clinical dimensions. Psychol Med 1993;23: 579-90.

43. Ebert D, Ebmeier KP. The role of the cingulate gyrus in depression: from functional anatomy to neurochemistry. Biol Psychiatry 1996;39:1044-50.

44. Drevets WC, Price JL, Simpson JR, Todd RD, Reich T, Vannier M, et al. Subgenual prefrontal cortex abnormalities in mood disorders. Nature 1997;386:824-27.

45. Keshavan MS, Kapur S, Pettegrew JW. Magnetic resonance spectroscopy in psychiatry: potential, pitfalls, and promises. Am J Psychiatry 1991;148:976-85.

46. Dager SR, Grant Steen RG. Applications of magnetic resonance spectroscopy to the investigation of neuropsychiatric disorders. Neuropsychopharmacology 1992;6:249-66.

47. Renshaw PF, Lafer B, Babb SM, Fava M, Stoll AL, Christensen JD, et al.. Basal ganglia choline levels in depression and response to fluoxetine treatment: an in vivo proton magnetic resonance spectroscopy study. Biol Psychiatry 1997;41:837-43.

48. Kato T, Takahashi S, Shioiri T, Inubushi T. Brain phosphorous metabolism in depressive disorders detected by phosphorus-31 magnetic resonance spectroscopy. J Affect Disord 1992;26:223-30.

49. Moore CM, Christensen JD, Lafer B, Fava M, Renshaw PF. Lower levels of nucleoside triphosphate in the basal ganglia of depressed subjects: a phosphorous-31 magnetic resonance spectroscopy study. Am J Psychiatry 1997; 154:116-18.

\section{Correspondência Beny Lafer / Homero Pinto Vallada Filho}

R. Dr. Ovídio Pires de Campos s/n. - São Paulo, SP. CEP: 05403-010, Tel/fax: + 5511 3064-3321 